\title{
Hypothesis
}

\section{The synthesis of Bcl-2 and other proteins in the neoplastic follicles of follicular lymphoma}

\author{
I A Lampert
}

The bcl-2 proto-oncogene was discovered at the chromosomal breakpoint of the $\mathrm{t}(14 ; 18)$ translocation found in human follicular lymphoma; $\mathrm{t}(14 ; 18)$ juxtaposes the bcl-2 gene from chromosome 18 with the immunoglobulin heavy chain (IGH) locus on chromosome $14 .^{1}$ This creates a bcl-2-IGH fusion gene that is markedly deregulated, resulting in the overproduction of bcl-2 RNA and protein. bcl-2 has the oncogenic function of blocking programmed cell death. Furthermore, bcl-2IGH transgenic mice overexpress the bcl-2 gene in lymphoid tissues and develop a polyclonal expansion of small resting B cells. ${ }^{2}$ These cells accumulate because they fail to die, demonstrating prolonged survival. However, these B cells can proceed to high grade lymphomas, suggesting that extended cell survival is tumorigenic. ${ }^{3}$

The germinal centre of the lymphoid follicle provides a microenvironment for the generation of memory B cells and plasma cells. After stimulation with antigen, $B$ cell blasts seed the primary follicle, which matures into a secondary germinal centre with well defined anatomical zones. ${ }^{4} \mathrm{Bcl}-2$ protein staining is intense in the follicular mantle zone. B lymphpocytes within the interfollicular regions are often positive. In striking contrast, most cells within the germinal centre are negative for Bcl-2. ${ }^{5}$ Centroblasts within the dark zone fail to stain for $\mathrm{Bcl}-2$, which is also absent from centrocytes in the basal portion of the light zone, laden with tingible body macrophages. Bcl-2 protein returns uniformly at low intensity within the more apical portion of each light zone, where residual antigen localised to the surface of follicular dendritic cells appears to select high affinity B cells. Most Bcl-2 positive cells coexpress the CD22 B cell marker but not the CD3 $\mathrm{T}$ cell marker.

There is considerable evidence to suggest that

Department of Histopathology, Ealing Hospital NHS Trust, Uxbridge Road, Southall, Middlesex UB13HW, UK

I A Lampert

Correspondence to: Dr Lambert.

Accepted for publication 30 June 1998 but also evidence of ongoing somatic mutation and antigen affinity selection.

In contrast to the benign follicle, the cells of most cases of follicular lymphoma are Bcl-2 positive. Because $85-90 \%{ }^{7}$ of cases of follicular lymphoma have the $t(14 ; 18)$ translocation, it has been assumed that this translocation causes excess stimulation of the bcl-2 gene and is therefore responsible for the Bcl-2 positivity of the neoplastic follicles.

However, there is evidence suggesting that this is not the case, namely:

- Many low grade B cell lymphomas such as chronic lymphocytic leukaemia (CLL) are $\mathrm{Bcl}-2$ positive without showing the $\mathrm{t}(14 ; 18)$ translocation. ${ }^{8}$

- There are cases of follicular lymphomas that do not show the $\mathrm{t}(14 ; 18)$ translocation, yet the neoplastic follicles are Bcl-2 positive. ${ }^{7}$

- Although all low grade follicular lymphomas are Bcl-2 positive, only $75 \%$ of high grade follicular lymphomas are Bcl-2 positive. ${ }^{9}$

- Within an individual lymphoma, there is a variability in Bcl-2 positivity, with the proliferating centroblasts being negative and the centrocytes positive. ${ }^{9}$

- If the $\mathrm{t}(14 ; 18)$ translocation was the explanation for the expression of the phenomenon, one would expect that in the neoplastic follicle there would be far more bcl-2 mRNA than in the benign follicles, which is not true. There is abundant bcl-2 mRNA as assessed by in situ hybridisation in the benign follicles, possibly more that is found in the neoplastic ones. ${ }^{10-12}$

- In cases where there are bcl-2 negative follicles and an interfollicular neoplastic infiltrate, there is a strange appearance of the negative follicles, contrasting with the positive interfollicular areas ( $\mathrm{P}$ Kluin, personal communication, 1998).

What makes this even less credible a story is the evidence that the phenomena that affect the expression of the bcl-2 gene in benign and neoplastic follicles affect several other proteins. Thus:

- The synthesis of several proteins is known to be downregulated when $\mathrm{B}$ cells move into the germinal centres-for example, CD44, L selectin, CD24, CD45RA, and several other uncharacterised proteins. ${ }^{13-15}$ Of these, CD24 and CD24RA are present in $60-80 \%$ of the ance of cells in the normal germinal centre and these cells are of B cell lineage. Analysis of the DNA shows not only clonal gene rearrangement 
neoplastic follicles and L selectin in about $20-30 \%$ of cases. None of these proteins is encoded on chromosomes 14 or 18 .

- The anomalous expression of bcl-2 mRNA has led to the view that the neoplastic process affects the control of protein synthesis in the germinal centre. We have studied the expression of the gene encoding CD24 in germinal centre and neoplastic follicles. In contrast, we have observed that in the normal germinal centre CD24 mRNA was absent, whereas in neoplastic follicles it was expressed strongly (unpublished data, 1998).

Thus, for this and probably the other proteins, there is no special effect on mRNA translation mechanisms in the germinal centre.

It has been shown that when follicular lymphoma affects the spleen then neoplastic cells, which are light chain restricted, populate the marginal zone as well as the other B cell areas of the white pulp. Neoplastic cells in the follicular portions of the white pulp are Bcl-2 positive, whereas those in the marginal zone are Bcl-2 negative. ${ }^{16}{ }^{17}$ Similarly, the marginal zone tumour cells are negative for both CD24 and CD45RA (unpublished data, 1998).

From this it is possible to conclude that the expression of bcl-2, CD24, and CD45RA is not fixed in follicular lymphoma cells, but is very much dependant on the environment and possibly the stimuli to which the tumour cells have been exposed.

This has led us to consider the proposition that the expression of the genes encoding Bcl-2 and these other proteins in neoplastic follicles is not so much a factor of an inherent property but an anomaly consequent on the failure of these structures to induce the downregulation of these genes. In support of this theory, in vitro studies have shown that if normal, resting $\mathrm{B}$ cells are stimulated with antibodies to the B cell receptor and a variety of cytokines they show such downregulation, including downregulation of the genes encoding CD24 and Bcl-2. ${ }^{18} 19$

A general form of support for this proposition comes from experimental studies with CLL cells in vitro. If the latter are stimulated to proliferate the synthesis of cytoplasmic Bcl-2 is downregulated. ${ }^{8}$

If this theory is correct, then there should be evidence to suggest that the neoplastic follicle is poorly formed and the neoplastic cells proliferating less actively than their benign counterparts. Such evidence does exist, namely:

- In follicular lymphoma, the follicular dendritic mesh is often very bizarre and "broken up" (for example, with staining for CD23).

- Electron microscopic studies show that the follicular dendritic cells are poorly formed with stunted dendrites. ${ }^{20}$ Acid cystein proteinase inhibitor and acetylcholinesterase have been shown to be present in the follicular dendritic cells of the normal germinal centre but are poorly expressed or absent in those in the neoplastic germinal centres. ${ }^{2122}$

- Follicular dendritic cells in normal germinal centres bind antigen and complement, this does not occur in neoplastic follicles. ${ }^{23}$

- In the normal germinal centre the cells show polarisation, with large centroblasts at one end and centrocytes at the other. The characteristic feature of neoplastic follicles in that this form of development is absent.

- The state of cell proliferation in the reactive germinal centre is in the order of $60 \%$, with cell proliferation in the dark zone being in the order of $100 \%$. It is of interest that the average state of proliferation in a follicular lymphoma $(\sim 20-30 \%)$ is that seen in the light zone of the normal germinal centre. ${ }^{24}$ Is it of importance that in the neoplastic follicles the proliferating centroblasts are often $\mathrm{Bcl}-2$ negative, in contrast to the non-proliferating centrocytes, which are $\mathrm{Bcl}-2$ positive.

Studying the distribution of Bcl-2 in the normal germinal centre provides a further possible explanation as to why follicular lymphomas are Bcl-2 positive. Korsmeyer's group found that the few Bcl-2 positive cells in the normal germinal centre were B cells, not T cells, as is so often stated. These cells were located in the subapical position in the germinal centre. In effect, this implies that as the germinal centre cells mature they reacquire the $\mathrm{Bcl}-2$ that they have lost. ${ }^{5}$ Is it possible that the phenotype of the cells in follicular lymphoma is that of the most mature type in the apical portion of the light zone, a consequence of their survival, escape from apoptosis, and subsequent maturation.

Staining for Bcl-2 is used extensively in the diagnosis of follicular lymphoma. Although it is very useful, anomalies have been encountered that are not predictable, nor easily explicable. This suggests that much has to be learned of the biology of this disease.

1 Cleary ML, Smith SD, Sklar J. Cloning and structural analysis of cDNAs for bcl-2 and a hybrid bcl2/ immunoglobulin transcript resulting from the $t(14 ; 18)$ translocation. Cell 1986;10;47:19-28.

2 McDonnell TJ, Deane N, Platt FM, et al. bcl-2immunoglobulin transgenic mice demonstrate extended B cell survival and follicular lymphoproliferation. Cell 1989; 57:79-88.

3 McDonnell TJ, Korsmeyer SJ. Progression from lymphoid hyperplasia to high-grade malignant lymphoma in mice transgenic for the $\mathrm{t}(\mathrm{t} 4 ; 18)$. Nature 1991;349:254-6.

4 MacLennan IC, Gray D. Antigen-driven selection of virgin and memory B cells. Immunol Rev 1986;91:61-85.

5 Hockenbery DM, Zutter M, Hickey W, et al. BCL2 protein is topographically restricted in tissues characterized by apoptotic cell death. Proc Natl Acad Sci USA 1991;88: 6961-5.

6 Harris NL, Jaffe ES, Stein H, et al. A revised EuropeanAmerican classification of lymphoid neoplasms: a proposal from the international lymphoma study group. Blood 1994; 84:1361-92.

7 Pezella F, Gatter KC, Mason DY, et al. bcl-2 protein expression in follicular lymphomas in the absence of $14 ; 18$ translocation. Lancet 1990;336:1510-1.

8 Caligaris-Cappio F, Gottardi D, Alfarano A, et al. The nature of the B lymphocyte in B-chronic lymphocytic leukemia. Blood Cells 1993;19:601-13.

9 Gaulard P, d'Agay MF, Peuchmaur M, et al. Expression of the bcl-2 gene product in follicular lymphoma. Am $\mathcal{F}$ Pathol 1992;140:1089-95.

10 Kondo E, Nakamura S, Onoue H, et al. Detection of bcl-2 protein and bcl-2 messenger RNA in normal and neoplastic lymphoid tissues by immunohistochemistry and in situ hybridization. Blood 1992;80:2044-51.

11 Chleq-Deschamps CM, LeBrun DP, Huie P, et al. Topographical dissociation of BCL-2 messenger RNA and protein expression in human lymphoid tissues. Blood 1993; 81:293-8.

12 Akagi T, Kondo E, Yoshino T. Expression of Bcl-2 protein and Bcl-2 mRNA in normal and neoplastic lymphoid tissues. Leuk Lymphoma 1994;13:81-7.

13 Pilkington GS, Rockman J, Quirk S, et al. Association of phenotype determined using monoclonal antibodies CDICD24 with histologic classification and karyotype in 180 non-Hodgkin's lymphomas. In: McMichael AJ, Beverley PCL, Cobbold S, et al., eds. Leucocyte typing III white cell differentiation antigens. Oxford: Oxford University Press, 1987:454-61. 
14 Paramithiotis E, Cooper MD. Memory B lymphocytes migrate to bone marrow in humans. Proc Natl Acad Sci USA 1997;94:208-12.

15 Kremmidiotis G, Zola $\mathrm{H}$. Changes in CD44 expression during B cell differentiation in the human tonsils. Cell Immunol 1995;161;147-57.

16 Schmid U, Cogliatti SB, Diss TC, et al. Monocytoid/ marginal zone B-cell differentiation in follicle centre cell lymphoma. Histopathology 1996;29:201-8.

17 Alkan S, Ross CW, Hanson CA, et al. Follicular lymphoma with involvement of the splenic marginal zone: a pitfall in the differential diagnosis of splenic marginal zone cell lymphoma. Hum Pathol 1996;27:503-6.

18 Galibert L, Burdin N, de Saint-Vis B, et al. CD40 and B cell antigen receptor dual triggering of resting lymphocyte turns on a partial germinal center phenotype. $\mathcal{F}$ Exp Med 1996;183:77-85.

19 Grimaitre M, Werner-Favre C, Kindler V, et al. Human naive $B$ cells cultured with EL-4 T cells mimic a germinal center-related B cell stage before generating plasma cells.
Concordant changes in Bcl-2 protein and messenger RNA levels. Eur F Immunol 1997;27:199-205.

20 Peters JPJ, Rademakers LEPM, De Boer RI, et al. Cellular composition of follicles of follicle centre cell lymphomas in relation to germinal centres of reactive lymph nodes. A morphometrical electromicroscopical study. F Pathol 1987; 153:233-44

21 Alavaikko M, Aine R, Rinne A, et al. Behaviour of dendritic reticulum cells possessing immunoreactive acid cysteine proteinase inhibitor in human lymphoid secondary follicles and in follicular-centre cell lymphomas. Int $\mathcal{F}$ Cancer 1985; 35:319-25.

22 Lampert IA, Van Norden S. Acetyl cholinesterase is expressed in the follicular dendritic cells of germinal centres: differences between normal and neoplastic follicles. F Pathol 1996;180:169-74.

23 Stein H, Gerdes J, Mason DY. The normal and malignant germinal centre. Clin Haematol 1992;11:531-58.

24 Cibill ML, Heryet K, Gatter KC, et al. The utility of Ki67 immunostaining, nuclear organizer region counting and morphology of follicular lymphomas. F Pathol 1989;158: 189-93. 